\title{
On a Whitham-Type Equation
}

Sergei SAKOVICH

Institute of Physics, National Academy of Sciences, 220072 Minsk, Belarus

E-mail:saks@tut.by

Received September 27, 2009, in final form November 05, 2009; Published online November 08, 2009

doi:10.3842/SIGMA.2009.101

\begin{abstract}
The Hunter-Saxton equation and the Gurevich-Zybin system are considered as two mutually non-equivalent representations of one and the same Whitham-type equation, and all their common solutions are obtained exactly.
\end{abstract}

Key words: nonlinear PDEs; transformations; general solutions

2000 Mathematics Subject Classification: 35Q58; 35C05

\section{Introduction}

The following Whitham-type equation

$$
u_{t}=2 u u_{x}-\partial_{x}^{-1} u_{x}^{2}
$$

was proposed recently by Prykarpatsky and Prytula in [1] as a model equation describing the short-wave perturbations in an abstract elastic one-dimensional medium with relaxation and spatial memory effects.

This equation (1), containing the ill-defined term $\partial_{x}^{-1} u_{x}^{2}$, was represented in [1] by the secondorder nonlinear partial differential equation

$$
u_{x t}=2 u u_{x x}+u_{x}^{2}
$$

and it was shown there that (2) is integrable in the sense of possessing a bi-Hamiltonian structure, an infinite hierarchy of conservation laws, and a Lax-type representation. Also, two finitedimensional reductions of (2) were obtained in [1], and they turned out to be some integrable by quadratures dynamical system, which could be useful for deriving wide classes of exact solutions of $(2)$.

A different representation of (1), the hydrodynamic-type system

$$
u_{t}=2 u u_{x}-v, \quad v_{t}=2 u v_{x},
$$

was proposed recently by Bogoliubov, Prykarpatsky, Gucwa, and Golenia in [2]. In the first equation of (3), the ill-defined term $\partial_{x}^{-1} u_{x}^{2}$ of (1) was replaced by the new variable $v$, while the time evolution of $v$ was determined by an additional first-order equation, the second equation of (3). It was announced in [2] that (3) is integrable in the sense of possessing a Lax-type representation. The system (3) was called in [2] an integrable regularization of the Whithamtype equation (1).

In the present paper, we consider the nonlinear PDEs (2) and (3) in Sections 2 and 3, respectively. We give the references which show that these equations (2) and (3), especially the first one of them, were known and quite well studied in the literature prior to $[1,2]$. We put a particular emphasis on the fact that the equations (2) and (3) can be completely solved by quadratures, and we refine the derivations of their general solutions, consistently following the 
way used for the Rabelo equations in [3]. We have the following reasons to re-derive the general solutions of (2) and (3). Firstly, the different derivations of the general solutions of (2), given in the literature, all overlooked the evident class of $x$-independent solutions. Secondly, the general solution of (3) can be expressed not only in a parametric form, known in the literature, but also in an implicit form. And thirdly, we need to get the results in uniform notations, for Section 4, where we make a comparison of the general solutions of (2) and (3), taking into account that these two representations of the Whitham-type equation (1) are not equivalent to each other, and obtain all common solutions of (2) and (3) exactly.

\section{The Hunter-Saxton equation}

The nonlinear equation (2) is far not novel. Up to a scale transformation of its variables, this is the celebrated Hunter-Saxton equation [4], sometimes referred to as the Hunter-Zheng equation [5]. The Hunter-Saxton equation has been studied in almost all respects, including its complete solvability by quadratures $[4,6,7,8]$, relationship with the Camassa-Holm equation and the Liouville equation [6, 7], bi-Hamiltonian formulation [5, 9], integrable finite-dimensional reductions [5, 10], global solution properties [11, 12], and geometric interpretations [13, 14], to mention only a few of numerous publications on this equation.

In our opinion, the most important feature of the Hunter-Saxton equation is the possibility to obtain its general solution in a closed form. This equation is linearizable [8], or C-integrable in the Calogero's terminology, but it also belongs to a subset of C-integrable equations whose general solutions can be expressed in a closed form. Such completely solvable equations of the Liouville equation's type differ from other C-integrable equations of the Burgers equation's type, and from the so-called S-integrable (completely integrable, or Lax integrable) equations of the sine-Gordon equation's type, in many respects. For example, the Liouville equation possesses a continuum of variational symmetries (hence, a continuum of nontrivial conservation laws) and several Lax-type representations which all turn out to be equivalent to conservation laws [15]. Leaving a study of such properties of the Hunter-Saxton equation for a separate publication, here we only concentrate on its general solution. The general solution of (2) can be obtained in a parametric form, in at least three different ways $[4,7,8]$. The derivation we give below is similar to the original one of [4], but differs from it by a more precise treatment of the arbitrariness of the transformation involved, in the spirit of $[3]$.

Making the transformation

$$
x=x(y, t), \quad x_{y} \neq 0, \quad u(x, t)=a(y, t),
$$

where the function $x(y, t)$ is initially not fixed, and using the identities

$$
\begin{aligned}
& u_{x}=\frac{a_{y}}{x_{y}}, \quad u_{t}=a_{t}-\frac{a_{y} x_{t}}{x_{y}}, \quad u_{x x}=\frac{a_{y y}}{x_{y}^{2}}-\frac{a_{y} x_{y y}}{x_{y}^{3}}, \\
& u_{x t}=\frac{a_{y t}}{x_{y}}-\frac{a_{y y} x_{t}+a_{y} x_{y t}}{x_{y}^{2}}+\frac{a_{y} x_{y y} x_{t}}{x_{y}^{3}},
\end{aligned}
$$

we bring the nonlinear equation (2) into the form

$$
a_{y t}+\frac{a_{y}^{2}}{x_{y}}=\partial_{y}\left(\frac{\left(x_{t}+2 a\right) a_{y}}{x_{y}}\right) .
$$

Now we see from (6) that it is expedient to fix the function $x(y, t)$ of the transformation (4) by the condition $x_{t}+2 a=0$, which brings the equation into a constant-characteristic form and 
considerably simplifies it. Doing this, we find that the transformation (4) with

$$
a=-\frac{1}{2} x_{t}
$$

relates the second-order equation (2) with the third-order equation

$$
x_{y t t}-\frac{x_{y t}^{2}}{2 x_{y}}=0
$$

which follows from (6) and (7).

Through the transformation (4) with (7), the general solution of the third-order equation (8) represents the general solution of the second-order equation (2) parametrically, with $y$ being the parameter. Note, however, that, according to the Cauchy-Kovalevskaya theorem [16], the general solution of (8) must contain three arbitrary functions of one variable, whereas the general solution of (2) must contain only two arbitrary functions of one variable. This redundant arbitrariness in $x(y, t)$, caused by the invariance of (8) with respect to an arbitrary transformation $y \mapsto Y(y)$ which has no effect on $u(x, t)$ of $(2)$, can be eliminated by the following normalization of the parameter $y$. We rewrite (8) in the form

$$
\partial_{t}\left(x_{y}^{-1 / 2} x_{y t}\right)=0
$$

integrate over $t$, and get

$$
x_{y}^{-1 / 2} x_{y t}=f(y),
$$

where $f(y)$ is an arbitrary function. For any nonzero function $f(y)$, we can set, without loss of generality, $f=2$ in (9) by an appropriate transformation $y \mapsto Y(y)$ which does not change the corresponding solutions of (2), where the value 2 is chosen for convenience only. The case of $f=0$ must be considered separately. Consequently, all solutions of the second-order equation (2) are represented parametrically by all solutions of the second-order equation (9) with $f=0$ and $f=2$ through the transformation (4) with (7).

The case of $f=0$ in (9) is $x_{y t}=0$, which immediately leads us through (7) and (5) to $a_{y}=0$ and $u_{x}=0$, that is, to the evident class of solutions

$$
u=\tau(t)
$$

of (2), with any function $\tau(t)$. In the case of $f=2$, we integrate (9) over $t$ and get

$$
x_{y}=(t+\phi(y))^{2},
$$

with any function $\phi(y)$. Then, integrating (11) over $y$ and using (7) and (4), we obtain the following class of solutions of (2), determined parametrically:

$$
\begin{aligned}
& x=y t^{2}+2 t \int \phi(y) d y+\int \phi(y)^{2} d y+\psi(t), \\
& u(x, t)=-y t-\int \phi(y) d y-\frac{1}{2} \psi^{\prime}(t),
\end{aligned}
$$

where $y$ is the parameter, $\phi(y)$ and $\psi(t)$ are arbitrary functions, and the prime denotes the derivative. The expressions (10) and (12) together constitute the general solution of the secondorder nonlinear partial differential equation (2).

Some words are due on the obtained general solution of (2). It follows from (12) that

$$
u_{x}=\frac{-1}{t+\phi(y)}
$$


According to this relation (13), the condition $u_{x} \neq 0$ is satisfied for any function $\phi(y)$, which proves that the class of solutions (12) does not cover solutions of the class (10). For some unknown reasons, only the parametric expressions (12) were called the general solution of (2) in $[4,7,8]$, whereas the solutions (10) were omitted there. Also, the relation (13) makes clear that all solutions of (2), except for those of the class (10), inevitably possess singularities of the type $u_{x}= \pm \infty$, when considered on the interval $-\infty<t<\infty$. The transformation (4), used for obtaining the general solution of (2), is applicable everywhere outside those singularities $u_{x}= \pm \infty$, because the condition $x_{y} \neq 0$ is satisfied due to (11). This inevitable presence of singularities in the solutions (12) was noticed in [4]. In the next section, we show that nontrivial solutions of the representation (3) of the Whitham-type equation (1) not necessarily contain blow-ups of derivatives.

\section{The Gurevich-Zybin system}

Proceeding to the hydrodynamic-type system (3), we note that this is the one-dimensional reduction of the Gurevich-Zybin system [17, 18] which can be completely solved by quadratures $[18,19]$. For an earlier appearance of (3) in plasma physics, one can consult Section 3 of [20]. In [21], a bi-Hamiltonian structure and a zero-curvature representation were found and studied for the system (3). Below we show how to obtain the general solution of (3) in an implicit form, following the way used in [3].

Applying the transformation

$$
x=x(y, t), \quad x_{y} \neq 0, \quad u(x, t)=a(y, t), \quad v(x, t)=b(y, t)
$$

to the system $(3)$, with $x(y, t)$ being not fixed initially, we obtain

$$
a_{t}-\frac{\left(x_{t}+2 a\right) a_{y}}{x_{y}}+b=0, \quad b_{t}-\frac{\left(x_{t}+2 a\right) b_{y}}{x_{y}}=0 .
$$

Then we fix the function $x(y, t)$ in (14) and (15) by the condition $x_{t}+2 a=0$, and thus get

$$
a=-\frac{1}{2} x_{t}, \quad b=\frac{1}{2} x_{t t}, \quad x_{t t t}=0,
$$

that is,

$$
x=\alpha(y) t^{2}+\beta(y) t+\gamma(y), \quad a=-\alpha(y) t-\frac{1}{2} \beta(y), \quad b=\alpha(y),
$$

where $\alpha(y), \beta(y), \gamma(y)$ are three arbitrary functions, of which at least one is non-constant due to $x_{y} \neq 0$. The expressions (14) and (16) represent the general solution of the system (3) parametrically, with $y$ being the parameter. An appropriate transformation $y \mapsto Y(y)$, which has no effect on solutions $u(x, t), v(x, t)$ of (3), may be used to fix any one of the three arbitrary functions in (16).

This parametric general solution of (3) can be expressed in an implicit form, as follows. When the function $\alpha(y)$ is non-constant, we replace $a(y, t)$ and $b(y, t)$ in $(16)$ by $u(x, t)$ and $v(x, t)$, respectively, then eliminate $y$ from the resulting expressions, and thus obtain

$$
x+v t^{2}+2 u t+\mu(v)=0, \quad u+v t+\nu(v)=0,
$$

where $\mu(v)$ and $\nu(v)$ are arbitrary functions (expressible in terms of the arbitrary functions $\alpha$, $\beta, \gamma)$. When $\alpha(y)$ is constant but $\beta(y)$ is not, we do the same and get

$$
x+\xi t^{2}+2 u t+\rho(u+\xi t)=0, \quad v=\xi,
$$


where $\rho$ is an arbitrary function of its argument, and $\xi$ is an arbitrary constant. When $\alpha(y)$ and $\beta(y)$ are constant but $\gamma(y)$ is not, we get

$$
u=\eta t+\zeta, \quad v=-\eta,
$$

where $\eta$ and $\zeta$ are arbitrary constants. These expressions (17)-(19) together constitute the general solution of the nonlinear system (3).

Unlike all nontrivial solutions of the Hunter-Saxton equation, some solutions of the GurevichZybin system (3), of the class (17), do not contain blow-ups of derivatives. Indeed, it follows from (17) that the expression for any derivative of $u$ or $v$ contains only some degree of the expression $t^{2}+2 t \nu^{\prime}(v)-\mu^{\prime}(v)$ in its denominator, for example,

$$
u_{x}=\frac{-t-\nu^{\prime}(v)}{t^{2}+2 t \nu^{\prime}(v)-\mu^{\prime}(v)}, \quad v_{x}=\frac{1}{t^{2}+2 t \nu^{\prime}(v)-\mu^{\prime}(v)},
$$

where the prime denotes the derivative. Clearly, it is possible to choose the functions $\mu$ and $\nu$ so that $t^{2}+2 t \nu^{\prime}(v)-\mu^{\prime}(v) \neq 0$ holds on the whole interval $-\infty<t<\infty$.

\section{Discussion}

The general solutions of the Hunter-Saxton equation (2) and the Gurevich-Zybin system (3) are quite different in their structure. The general solution of (2) is given in the parametric form (12), except for the explicit solutions (10). The general solution of (3) is given in the implicit form (17) and (18), except for the explicit solutions (19). From this point of view, the Hunter-Saxton equation (2) and the Gurevich-Zybin system (3) are very similar to the expRabelo equation $u_{x t}=\exp u-(\exp u)_{x x}$ and the quadratic Rabelo equation $u_{x t}=1+\frac{1}{2}\left(u^{2}\right)_{x x}$, respectively [3].

The nonlinear PDEs (2) and (3) were considered in [1, 2] as two well-defined representations of the Whitham-type equation (1) which itself contains the ill-defined term $\partial_{x}^{-1} u_{x}^{2}$. Evidently, these two representations are not equivalent to each other. The Gurevich-Zybin system (3) can be re-written as the second-order equation

$$
u_{t t}-4 u u_{x t}+4 u^{2} u_{x x}-2 u_{x} u_{t}+4 u u_{x}^{2}=0
$$

for $u(x, t)$ with the definition $v=2 u u_{x}-u_{t}$ for $v(x, t)$, and this second-order equation (20) differs from the Hunter-Saxton equation (2). For this reason, one may wonder whether the PDEs (2) and (3) have any common nontrivial solutions at all.

It can be found easily that the compatibility condition for the equations (2) and (20) is

$$
u_{t t}=4 u^{2} u_{x x}+2 u_{x} u_{t} .
$$

Alternatively, in the variables $u$ and $v$, the compatibility condition for the PDEs (2) and (3) is

$$
v_{x}=u_{x}^{2} .
$$

One can find all common solutions of (2) and (3) by applying the condition (21) to the general solution (10) and (12) of the Hunter-Saxton equation, or, alternatively, by applying the condition (22) to the general solution (17)-(19) of the Gurevich-Zybin system. Using the condition (21), we get

$$
\tau^{\prime \prime}=0
$$


from (10), and

$$
\psi^{\prime \prime \prime}=0
$$

from (12). Using the condition (22), we get

$$
\mu^{\prime}+\nu^{\prime 2}=0
$$

from (17), while (18) does not satisfy (22), and (19) satisfies (22) identically. It is quite obvious that (10) with (23) is equivalent to (19), and that (12) with (24) is equivalent to (17) with (25).

Thus, summarizing the result in a nonrigourous way, we can say that the degree of arbitrariness of common nontrivial solutions of the Hunter-Saxton equation (2) and the Gurevich-Zybin system (3) is one arbitrary function of one variable.

\section{Acknowledgement}

The author is deeply grateful to Professor E.V. Ferapontov and Professor M.V. Pavlov for pointing out the origin of the system (3), to the referees for their useful suggestions, and to the Max-Planck-Institut für Mathematik for hospitality and support.

\section{References}

[1] Prykarpatsky A.K., Prytula M.M., The gradient-holonomic integrability analysis of a Whitham-type nonlinear dynamical model for a relaxing medium with spatial memory, Nonlinearity 19 (2006), 2115-2122.

[2] Bogoliubov N.N. Jr., Prykarpatsky A.K., Gucwa I., Golenia J., Analytical properties of an OstrovskyWhitham type dynamical system for a relaxing medium with spatial memory and its integrable regularization, arXiv:0902.4395.

[3] Sakovich A., Sakovich S., On transformations of the Rabelo equations, SIGMA 3 (2007), 086, 8 pages, arXiv:0705.2889.

[4] Hunter J.K., Saxton R., Dynamics of director fields, SIAM J. Appl. Math. 51 (1991), 1498-1521.

[5] Hunter J.K., Zheng Y., On a completely integrable nonlinear hyperbolic variational equation, Phys. D 79 (1994), 361-386.

[6] Dai H.-H., Pavlov M., Transformations for the Camassa-Holm equation, its high-frequency limit and the Sinh-Gordon equation, J. Phys. Soc. Japan 67 (1998), 3655-3657.

[7] Pavlov M.V., The Calogero equation and Liouville-type equations, Theoret. and Math. Phys. 128 (2001), 927-932, nlin.SI/0101034.

[8] Morozov O.I., Contact equivalence of the generalized Hunter-Saxton equation and the Euler-Poisson equation, math-ph/0406016.

[9] Olver P.J., Rosenau P., Tri-Hamiltonian duality between solitons and solitary-wave solutions having compact support, Phys. Rev. E 53 (1996), 1900-1906.

[10] Beals R., Sattinger D.H., Szmigielski J., Inverse scattering solutions of the Hunter-Saxton equation, Appl. Anal. 78 (2001), 255-269.

[11] Hunter J.K., Zheng Y.X., On a nonlinear hyperbolic variational equation. I. Global existence of weak solutions, Arch. Rational Mech. Anal. 129 (1995), 305-353.

[12] Bressan A., Constantin A., Global solutions of the Hunter-Saxton equation, SIAM J. Math. Anal. 37 (2005), 996-1026, math.AP/0502059.

[13] Reyes E.G., The soliton content of the Camassa-Holm and Hunter-Saxton equations, in Proceedinds of Fifth International Conference "Symmetry in Nonlinear Mathematical Physics" (July 9-15, 2001, Kyiv), Editors A.G. Nikitin, V.M. Boyko and R.O. Popovych, Proceedings of Institute of Mathematics, Kyiv 43 (2002), Part 1, 201-208.

[14] Lenells J., The Hunter-Saxton equation: a geometric approach, SIAM J. Math. Anal. 40 (2008), $266-277$. 
[15] Sakovich S.Yu., On conservation laws and zero-curvature representations of the Liouville equation, J. Phys. A: Math. Gen. 27 (1994), L125-L129.

[16] Olver P.J., Applications of Lie groups to differential equations, 2nd ed., Graduate Texts in Mathematics, Vol. 107, Springer-Verlag, New York, 1993.

[17] Gurevich A.V., Zybin K.P., Nondissipative gravitational turbulence, Soviet Phys. JETP 67 (1988), 1-12.

[18] Gurevich A.V., Zybin K.P., Large-scale structure of the Universe. Analytic theory, Soviet Phys. Usp. 38 (1995), 687-722.

[19] Pavlov M.V., The Gurevich-Zybin system, J. Phys. A: Math. Gen. 38 (2005), 3823-3840, nlin.SI/0412072.

[20] Davidson R.C., Methods in nonlinear plasma theory, Academic Press, New York, 1972.

[21] Brunelli J.C., Das A., On an integrable hierarchy derived from the isentropic gas dynamics, J. Math. Phys. 45 (2004), 2633-2645, nlin.SI/0401009. 